Fault Detection and Diagnosis for General Discrete-time Stochastic Systems Using Output Probability Density Estimation.

Skaf, Zakwan and Al-Bayati, Ahmad and Wang, Hong 2011

MIMS EPrint: 2012.12

Manchester Institute for Mathematical Sciences

School of Mathematics

The University of Manchester

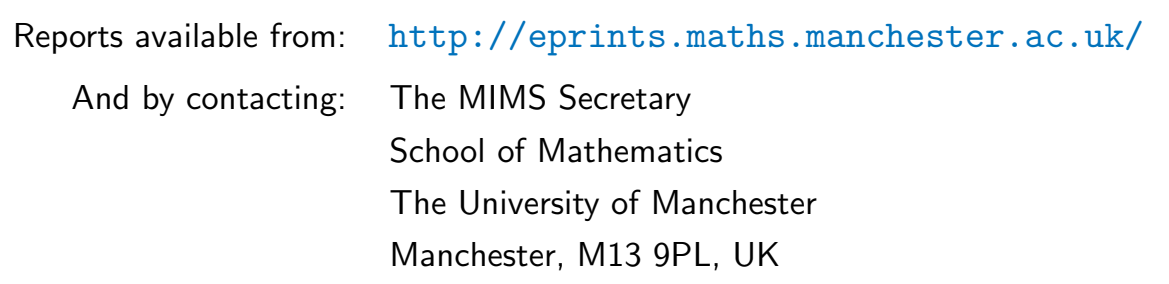




\title{
Fault Detection and Diagnosis for General Discrete-time Stochastic Systems Using Output Probability Density Estimation.
}

\author{
Zakwan Skaf, Ahmad AI-Bayati and Hong Wang
}

\begin{abstract}
A new approach of fault detection and diagnosis (FDD) for general stochastic systems in discrete-time is studied. Our work on this problem is motivated by the fact that most of the nonlinear control laws are implemented as digital controllers in reality. Different from the formulation of classical FDD problem, it is supposed that the measured information for the FDD is the probability density functions (PDFs) of the system output rather than its measured value. A radial basis function (RBF) neural network technique is proposed so that the output PDFs can be formulated in terms of the dynamic weighting of the RBFs neural network. Feasible criteria to detect and diagnose the system fault are provided by using linear matrix inequality (LMI) techniques. An illustrated example is included to demonstrate the efficiency of the proposed algorithm, and satisfactory results are obtained.
\end{abstract}

\section{INTRODUCTION}

Under the assumption that the random variables or the noise in the stochastic system are subject to Gaussian processes, the following approaches have been widely applied in theoretical studies: minimum variance control [1], whose purpose is minimizing the variations in the controlled system outputs or tracking errors, linear optimal control [2], linear quadratic martingale control [3], and stochastic control for systems with Markovian jump parameters [4]. In all these methods, the targets are the mean and variance of the output. However, this assumption may not hold in some applications. For example, many variables in the paper-making systems do not obey Gaussian distributions [5], [6]. Therefore, a new measure of randomness, called the PDF control, should be employed for general stochastic systems with non-Gaussian variables [5]. In PDF control problems, the control objective is to design a control signal so that the PDF shape of the output variable follows a desired distribution.

There are many stochastic systems in practice whose outputs are the PDF of the system output[5], rather than the actual output values. For such cases, the measured output PDFs can be used as an output for the feedback control. Such types of stochastic systems are called Stochastic Distribution Control (SDC) systems [5]. Practical examples of SDC

Z. Skaf is with Control Systems Center, School of Electrical and Electronic Engineering, Sackville Street Building,University of Manchester, Manchester, M60 1QD, UK Zakwan.Skaf@manchester.ac.uk

A. AI-Bayati is with Control Systems Center, School of Electrical and Electronic Engineering, Sackville Street Building,University of Manchester, Manchester, M60 1QD, UK Ahmad.Al-Bayati@postgrad.manchester.ac.uk

H. Wang is with Control Systems Center, School of Electrical and Electronic Engineering, Sackville Street Building,University of Manchester, Manchester, M60 1QD, UK Hong.Wang@manchester.ac.uk systems in industrial applications include: Molecular weight distribution control [7], [8], combustion flame distribution processes [9], [10], particle size distribution control in polymerisation and powder processing industries [11], [12], and the wet-end of paper-making [5].

SDC was originally developed by Professor Hong Wang in 1996, when he considered a number of challenging paper machine modelling and control problems [5]. The process and the control were presented in a PDF form. As such, the purpose of the controller design was to obtain the PDF of the controller so that the closed-loop PDF would follow the pre-specified PDF. Since then, rapid developments have been made and introduced in different control applications [13]. The most exciting PDF control approaches are based on the B-spline model. Four types of model have been used in PDF control strategies, namely: The linear B-spline model [14], the square-root B-spline model [15], the rational B-spline model [16], and the rational square-root B-spline model [17]. Moreover, multi-layer perception (MLP) neural network models have been applied to the shape control for the output PDFs [18]. Recently, a RBF neural network has been used to approximate the output PDF of the system [19]. In this work, we have used RBFs instead of B-Splines which help generalize the output PDF expression and overcome the problems with B-spline-based functional approximations.

Many effective FDD strategies have been developed by researchers in the last several decades to cover various types of faulty systems [20], [21], [22]. For stochastic systems, many significant schemes have been introduced and applied to practical process successfully. In general, the following approaches have been widely applied and developed for this problem: filter-or observer-based approaches [22], [23], identification-based approaches [24], and static approaches based likelihood approaches [25]. For dynamic stochastic systems, the filter based FDD approaches, have been presented as an effective way for Gaussian variables in stochastic systems. However, in many practical process, nonGaussian variables exist in many stochastic systems. In this case, the filter-based FDD for non-Gaussian stochastic systems may be incapable. Therefore, for non-Gaussian stochastic systems, a new FDD approach has been established by using output distribution function for general stochastic systems in [22], where the dynamical system was supposed to be a precise linear model and the design algorithm required some technical conditions that were hard to verify. That work was motivated by the retention system of the paper making process, where the system output is replaced by the measured 
output PDFs to generate the residual of the filter [22]. The residual signal is calculated via the use of either the weighted integration or the integration of the square of the difference between the measured and the estimated PDFs. This method was the first attempt focussing on the application of the PDF model. However, there was a criticism that the used linear B-spline model cannot guarantee the output PDF of the model is positive [23], [26]. Subsequently, an improved design approach has been applied for the general stochastic system by using a square root B-spline model and nonlinear filter design [26].

\section{PROBLEM FORMULATION}

In this section, this approach is different from the results in [26], a discrete-time RBF neural networks square root model is introduced to approximate the output PDFs, and then formulate a discrete-time nonlinear model for the weighting vectors.

Consider $u(k) \in R^{r}$ as the input of a discrete-time dynamic stochastic system, $y(k) \in[a, b]$ as the output, and $F(k)$ as the fault. At sample time k, $y(k)$ can be described by its PDF $\gamma(y, u(k))$, which is defined by

$$
P(a \leq y(k)<\xi)=\int_{a}^{\xi} \gamma(y, u(k), F(k)) d y
$$

where $P(a \leq y(k)<\xi, u(k))$ denotes the probability of output variable $y$ lying between $a$ and $\xi$ when the control is applied to the system.

It is assumed that the PDF is measurable and defined on a known interval $[a, b]$. The well-known RBF neural networks can be used to approximate the square root of the output $\mathrm{PDF}$ as follows [5].

$$
\begin{aligned}
\sqrt{\gamma(y, u(k), F(k))} & =R(y) V(k)+r_{n}(y) h(V(k)) \\
& +\omega(y, u(k), F(k))
\end{aligned}
$$

where $\gamma(y, u(k), F(k))$ is the output measured PDF.

$$
\begin{aligned}
R(y) & =\left[r_{1}(y), r_{2}(y), \ldots \ldots, r_{n-1}(y)\right] \\
V(k) & =\left[v_{1}(k), v_{2}(k), \ldots \ldots, v_{n-1}(k)\right]^{T} \\
\Lambda_{4} & =\Lambda_{2}^{T} \Lambda_{2}-\Lambda_{3} \Lambda_{1}, \Lambda_{1}=\int_{a}^{b} R^{T}(y) R(y) d y \\
\Lambda_{2} & =\int_{a}^{b} R^{T}(y) r_{n}(y) d y, \Lambda_{3}=\int_{a}^{b} r_{n}^{2}(y) d y
\end{aligned}
$$

and

$$
h(V(k))=\frac{1}{\Lambda_{3}}\left(-\Lambda_{2} V(k)+\sqrt{V^{T}(k) \Lambda_{4} V(k)}\right)
$$

$\mathrm{F}(\mathrm{k})$ is supposed to be an actuator fault, to be diagnosed and compensated. Term $\omega(y, u(k), F(k))$ represents the model uncertainties or the error term on the approximation of PDFs. In addition, $\omega(y, u(k), F(k))$ must satisfy the following condition [5]:

$$
|\omega(y, u(k), F(k))| \leq \delta
$$

where $\delta>0$ is a known positive constant. In (1), $R(y)$ and $V(k)$ are the activation function and weight element corresponding to RBF neural network used for PDF modelling, respectively. Similar to [27], [28], the RBF activation functions are chosen as of Gaussian shapes and expressed as follows.

$$
r_{l}(y)=\exp \left(-\frac{\left(y_{j}-\mu_{l}\right)^{2}}{2 \sigma_{l}^{2}}\right)
$$

where $\mu_{l}, \sigma_{l}$ are the centres and widths of the RBF basis functions, respectively. In (3), the nonlinear function $h(V(k))$ should satisfy the following Lipschitz condition for any $V_{1}(k), V_{2}(k)$ and a known matrix $U_{1}$.

$$
\left\|h\left(V_{1}(k)\right)-h\left(V_{2}(k)\right)\right\| \leq\left\|U_{1}\left(V_{1}(k)-V_{2}(k)\right)\right\|
$$

\section{A. Nonlinear Dynamic Weight Model}

In many cases, the dynamic relation between the input and the output PDFs can be transformed into dynamic relation between the control input and the weights of the RBFs neural network approximation to the output PDFs. In this section, the following discrete-time nonlinear weighting model will be used

$$
\begin{aligned}
x(k+1) & =A x(k)+B u(k)+G g(x(k))+D F(k) \\
V(k) & =E x(k)
\end{aligned}
$$

where $x(k) \in R^{n}$ is the state vector, and $u(k) \in R^{r}$ is the measurable input vector. Moreover, $A, B, G, D$ and $E$ represent the identified coefficient matrices of the weight system with suitable dimensions. $g(x(k))$ is a nonlinear vector function that stands for the nonlinear dynamics of the model, and is supposed to satisfy $g(0)=0$, and the following Lipschitz condition.

$$
\left\|g\left(x_{1}(k)\right)-g\left(x_{2}(k)\right)\right\| \leq\left\|U_{2}\left(x_{1}(k)-x_{2}(k)\right)\right\|
$$

for any $x_{1}(k)$ and $x_{2}(k)$, where $U_{2}$ is a known matrix. $F(k)$ is an actuator fault to be estimated and rejected. With model (6), equation (1) can be written as a nonlinear function of $x(k)$ as follows:

$$
\begin{aligned}
\sqrt{\gamma(y, u(k), F(k))} & =R(y) \operatorname{Ex}(k)+r_{n}(y) h(\operatorname{Ex}(k)) \\
& +\omega(y, u(k), F(k))
\end{aligned}
$$

Different from the models considered in [26], [22], the proposed discrete-time square root RBFNN model is more practical and better suited to digital control.

\section{FAULT DETECTION}

In order to detect the fault based on the changes of PDFs, the following nonlinear observer is considered:

$$
\begin{aligned}
& \hat{x}(k+1)=A \hat{x}(k)+B u(k)+G g(\hat{x}(k))+L \epsilon(k) \\
& \epsilon(t)=\int_{a}^{b} \mu(y)(\sqrt{\gamma(y, u(k, F))}-\sqrt{\hat{\gamma}(y, u(k))}) d y \\
& \sqrt{\hat{\gamma}(y, u(k))}=R(y) E \hat{x}(k)+h(E \hat{x}(k)) r_{n}(y)
\end{aligned}
$$

where $\hat{x}(k) \in R^{n}$ is the estimated state, $L \in R^{n \times p}$ is the filter gain to be determined. Residual $\epsilon(k)$ is formulated as an integral of the difference between the measured PDFs and the estimated ones, where, $\mu(y) \in R^{p \times 1}$ is a pre-specified weighting vector. 
Denoting the estimation error as $e(k)=x(k)-\hat{x}(k)$, the dynamic of the estimation error will be expressed as

$$
\begin{aligned}
e(k+1) & =\left(A-L \Gamma_{1}\right) e(k)+[G g(x(k))-G g(\hat{x}(k))] \\
& -L \Gamma_{2}[h(E x(k))-h(E \hat{x}(k))] \\
& -L \Delta(k)+D F(k)
\end{aligned}
$$

where

$$
\begin{aligned}
\Gamma_{1} & =\int_{a}^{b} \mu(y) R(y) E d y \\
\Gamma_{2} & =\int_{a}^{b} \mu(y) r_{n}(y) d y \\
\Delta(k) & =\int_{a}^{b} \mu(y) \omega(y, u(k)) d y
\end{aligned}
$$

It can be seen that

$$
\epsilon(k)=\Gamma_{1} e(k)+\Gamma_{2}[h(E x(k))-h(E \hat{x}(k))]+\Delta(k)
$$

From $|\omega(y, u(k), F)| \leq \delta$, it can be verified that

$$
\|\Delta(k)\|=\left\|\int_{a}^{b} \mu(y) \omega(y, u(k)) d y\right\| \leq \tilde{\delta}
$$

where $\tilde{\delta}=\delta\left\|\int_{a}^{b} \mu(y) d y\right\|$.

Theorem 1. For the parameter $\lambda_{i}>0(i=1,2)$, if there exit matrices $P>0$, and $R$ satisfying

$$
\Psi=\left[\begin{array}{cccc}
M_{1} & 0 & 0 & A^{T} P-\Gamma_{1}^{T} R^{T} \\
0 & -\lambda_{2}^{2} I & 0 & G^{T} P \\
0 & 0 & -\lambda_{1}^{2} I & \Gamma_{2}^{T} R^{T} \\
P A-R \Gamma_{1} & P G & -R \Gamma_{2} & -P
\end{array}\right]<0
$$

$M_{1}=-P+\lambda_{2}^{2} U_{2}^{T} U_{2}+\lambda_{1}^{2} E^{T} U_{1}^{T} U_{1} E$

then in the absence of fault, the error dynamic system with gain $L=P^{-1} R$ is stable and the error satisfies $\lim _{k \rightarrow \infty} e(k)=0$

Proof : For this purpose, the following Lyapunov function is considered.

$$
\begin{aligned}
\Phi(k) & =e^{T}(k) P e(k) \\
& +\lambda_{1}^{2} \sum_{i=1}^{k-1}\left[\left\|U_{1} E e(i)\right\|^{2}-\| h(E x(i))-h\left(E \hat{x}(i) \|^{2}\right]\right. \\
& +\lambda_{2}^{2} \sum_{i=1}^{k-1}\left[\left\|U_{2} e(i)\right\|^{2}-\| g(x(i))-g\left(\hat{x}(i) \|^{2}\right]\right.
\end{aligned}
$$

In the absence of $F(k)$, along with (10) it can be verfied that

$$
\begin{aligned}
\Delta \Phi & =\Phi(k+1)-\Phi(k) \\
& =e^{T}(k+1) P e(k+1)-e^{T}(k) P e(k) \\
& +\lambda_{1}^{2}\left[\left\|U_{1} E e(k)\right\|^{2}-\| h(E x(k))-h\left(E \hat{x}(k) \|^{2}\right]\right. \\
& +\lambda_{2}^{2}\left[\left\|U_{2} e(k)\right\|^{2}-\| g(x(k))-g\left(\hat{x}(k) \|^{2}\right]\right. \\
& =S_{k}^{T} \Psi_{1} S_{k}+2 S_{k}^{T}\left[\begin{array}{c}
-\left(A-L \Gamma_{1}\right)^{T} P L \\
-G^{T} P L \\
\Gamma_{2}^{T} L^{T} P L
\end{array}\right] \Delta(k) \\
& +\Delta^{T}(k) L^{T} P L \Delta(k)<0
\end{aligned}
$$

where

$$
\begin{aligned}
& \Psi_{1}=\left[\begin{array}{ccc}
\Psi_{2} & \left(A-L \Gamma_{1}\right)^{T} P G & -\left(A-L \Gamma_{1}\right)^{T} P L \Gamma_{2} \\
* & -\lambda_{2}^{2} I+G^{T} P G & -G^{T} P L \Gamma_{2} \\
* & * & -\lambda_{1}^{2} I+\Gamma_{2}^{T} L^{T} P L \Gamma_{2}
\end{array}\right] \\
& S_{k}^{T}= {\left[e^{T}(k),\left(g(x(k))-g(\hat{x}(k))^{T},\left(h(E x(k))-h(E \hat{x}(k))^{T}\right]\right.\right.} \\
& \Psi_{2}=\left(A-L \Gamma_{1}\right)^{T} P\left(A-L \Gamma_{1}\right)+M_{1}
\end{aligned}
$$

Denote $R=P L$, it can be seen that

$$
\begin{aligned}
\Delta \Phi & =S_{k}^{T} \Psi_{1} S_{k}+2 S_{k}^{T}\left[\begin{array}{c}
-\left(A-L \Gamma_{1}\right)^{T} R \\
-G^{T} R \\
\Gamma_{2}^{T} R^{T} P^{-1} R
\end{array}\right] \Delta(k) \\
& +\Delta^{T}(k) R^{T} P^{-1} R \Delta(k)<0
\end{aligned}
$$

By using the Schur complement formula, (17) can be further reduced to

$$
\Psi_{5}=\left[\begin{array}{ll}
\Psi_{3} & \Psi_{4}^{T} \\
\Psi_{4} & -P
\end{array}\right]<0
$$

where

$$
\Psi_{3}=\left[\begin{array}{ccc}
-P+\lambda_{2}^{2} U_{2}^{T} U_{2}+\lambda_{1}^{2} E^{T} U_{1}^{T} U_{1} E & 0 & 0 \\
* & -\lambda_{2}^{2} I & 0 \\
* & * & -\lambda_{1}^{2} I
\end{array}\right]
$$

and

$$
\Psi_{4}=\left[\begin{array}{lll}
P A-R \Gamma_{1} & P G & -R \Gamma_{2}
\end{array}\right]
$$

which is equivalent to (14). If (14) holds, a positive scalar exists $\varrho$ so that $\Psi \leq-\varrho I$. Thus, it can be seen that

$$
\begin{aligned}
\Delta \Phi & \leq-\varrho\left\|S_{k}\right\|^{2}-2\left\|\tilde{A}^{T} R\right\|\|\Delta(k)\|\left\|S_{k}\right\| \\
& +\|\Delta(k)\|^{2}\left\|R^{T} P^{-1} R\right\| \\
& \leq-\varrho\left\|S_{k}\right\|^{2}-2 \tilde{\delta}\left\|\tilde{A}^{T} R\right\|\left\|S_{k}\right\|+\tilde{\delta}^{2}\left\|R^{T} P^{-1} R\right\|
\end{aligned}
$$

where

$$
\tilde{A}=\left[\begin{array}{lll}
A-L \Gamma_{1} & P G & -R \Gamma_{2}
\end{array}\right]
$$

it can be shown that

$$
\left\|S_{k}\right\| \geq \tilde{\delta} \varrho^{-1}\left(\left\|\tilde{A}^{T} R\right\|+\sqrt{\left\|\tilde{A}^{T} R\right\|^{2}+\varrho\left\|R^{T} P^{-1} R\right\|}\right)
$$

which implies

$$
\begin{aligned}
\left\|S_{k}\right\| \leq & \max \left\{\left\|S_{k}(1)\right\|, \tilde{\delta} \varrho^{-1}\left(\left\|\tilde{A}^{T} R\right\|\right.\right. \\
& \left.+\sqrt{\left\|\tilde{A}^{T} R\right\|^{2}+\varrho\left\|R^{T} P^{-1} R\right\|}\right)
\end{aligned}
$$

This means that the error system in (10) is asymptotically stable under $\Psi<0$. Because $\lim _{k \rightarrow \infty} e(k)=0$, equations (5) and (7) guarantee that $\lim _{k \rightarrow \infty}(h(E x(k))-h(E \hat{x}(k)))=0$ and $\lim _{k \rightarrow \infty}(g(x(k))-g(\hat{x}(k)))=0$, which implies that $\lim _{k \rightarrow \infty} \Delta \Phi(k)=0$. 


\section{FAULT DIAGNOSIS}

Once the fault is detected, the fault value must be estimated. For this purpose, the following observer is considered:

$$
\begin{gathered}
\hat{x}(k+1)=A \hat{x}(k)+B u(k)+G g(\hat{x}(k))+L \epsilon(k)+D \hat{F}(k) \\
\sqrt{\hat{\gamma}(y, u(k))}=R(y) E \hat{x}(k)+r_{n}(y) h(E \hat{x}(k)) \\
\hat{F}(k+1)=-\Upsilon_{1} \hat{F}(k)+\Upsilon_{2} \epsilon(k)
\end{gathered}
$$

where $\hat{F}(k)$ is the estimation of $F(k) . \Upsilon_{1}>0$ and $\Upsilon_{2}$ are the learning operators to be determined together with $L$ by the diagnosis algorithm in (27). Denoting $\bar{F}(k)=F(k)-\hat{F}(k)$ and $e(k)=x(k)-\hat{x}(k)$. The dynamic of the estimation error will be expressed as

$$
\begin{aligned}
e(k+1) & =\left(A-L \Gamma_{1}\right) e(k)+[G g(x(k))-G g(\hat{x}(k))] \\
& -L \Gamma_{2}[h(E x(k))-h(E \hat{x}(k))]-L \Delta(k)+D \bar{F}(k)
\end{aligned}
$$

and

$$
\begin{aligned}
\bar{F}(k+1) & =F(k+1)-\hat{F}(k-1) \\
& =F(k+1)+\Upsilon_{1} \hat{F}(k)-\Upsilon_{2} \epsilon(k) \\
& =F(k+1)-\Upsilon_{1} F(k)+\Upsilon_{1} \bar{F}(k)-\Upsilon_{2} \epsilon(k) \\
& =\Delta F(k)+\Upsilon_{1} \bar{F}(k)-\Upsilon_{2} \Gamma_{1} e(k)-\Upsilon_{2} \Delta(k) \\
& -\Upsilon_{2} \Gamma_{2}[h(E x(k))-h(E \hat{x}(k))]
\end{aligned}
$$

where $\Delta F(k)=F(k+1)-\Upsilon_{1} F(k)$

Theorem 2. For the parameter $\lambda_{i}>0(i=1,2)$, if there exit matrices $P>0, R$, and $\Upsilon_{i}>0(i=1,2)$ satisfying

$$
\bar{\Psi}=\left[\begin{array}{ccccc}
\bar{\Psi}_{1} & 0 & M_{2} & M_{3} & \left(A-L \Gamma_{1}\right)^{T} P \\
* & -\lambda_{2}^{2} I & 0 & 0 & G^{T} P \\
* & * & M_{4} & M_{5} & \Gamma_{2}^{T} R^{T} \\
* & * & * & M_{6} & D^{T} P \\
* & * & * & * & -P
\end{array}\right]<0
$$

where

$$
\begin{aligned}
\bar{\Psi}_{1} & =-P+\lambda_{2}^{2} U_{2}^{T} U_{2}+\lambda_{1}^{2} E^{T} U_{1}^{T} U_{1} E+\Gamma_{1}^{T} \Upsilon_{2}^{T} \Upsilon_{2} \Gamma_{1} \\
M_{2} & =\Gamma_{1}^{T} \Upsilon_{2}^{T} \Upsilon_{2} \Gamma_{2}, M_{3}=\Gamma_{1}^{T} \Upsilon_{2}^{T} \Upsilon_{1} \\
M_{4} & =-\lambda_{1}^{2} I+\Gamma_{2}^{T} \Upsilon_{2}^{T} \Upsilon_{2} \Gamma_{2}, M_{5}=-\Gamma_{2}^{T} \Upsilon_{2}^{T} \Upsilon_{1} \\
M_{6} & =-I+\Upsilon_{1}^{T} \Upsilon_{1}
\end{aligned}
$$

then the filtering gain $L=P^{-1} R$, the error dynamic system is stable and the error satisfies

$$
\left\|\bar{S}_{k}\right\| \leq \max \left\{\left\|\bar{S}_{k}(1)\right\|, \varrho_{1}^{-1}\left(\left\|\Xi_{1}\right\|+\sqrt{\left\|\Xi_{1}\right\|^{2}+\varrho_{1}\left\|\Xi_{2}\right\|}\right)\right\}_{(31)}
$$

Proof : For this purpose, the following Lyapunov function is considered.

$$
\begin{aligned}
\Phi_{1}(k) & =e^{T}(k) P e(k)+\bar{F}^{T}(k) \bar{F}(k) \\
& \lambda_{1}^{2} \sum_{i=1}^{k-1}\left[\left\|U_{1} E e(i)\right\|^{2}-\| h(E x(i))-h\left(E \hat{x}(i) \|^{2}\right]\right. \\
& +\lambda_{2}^{2} \sum_{i=1}^{k-1}\left[\left\|U_{2} e(i)\right\|^{2}-\| g(x(i))-g\left(\hat{x}(i) \|^{2}\right]\right.
\end{aligned}
$$

It can be verfied that

$$
\begin{aligned}
\Delta \Phi_{1} & =\Phi_{1}(k+1)-\Phi_{1}(k) \\
& =e^{T}(k+1) P e(k+1)+\bar{F}^{T}(k+1) \bar{F}(k+1) \\
& -e^{T}(k) P e(k)-\bar{F}^{T}(k) \bar{F}(k) \\
& +\lambda_{1}^{2}\left[\left\|U_{1} E e(k)\right\|^{2}-\| h(E x(k))-h\left(E \hat{x}(k) \|^{2}\right]\right. \\
& +\lambda_{2}^{2}\left[\left\|U_{2} e(k)\right\|^{2}-\| g(x(k))-g\left(\hat{x}(k) \|^{2}\right]\right. \\
& =\bar{S}_{k}^{T} \bar{\Psi}_{2} \bar{S}_{k}+\Delta F^{T}(k) \Delta F(k)-2 \Delta F^{T}(k) \Upsilon_{2} \Delta(k) \\
& +\Delta^{T}(k)\left(L^{T} P L+\Upsilon_{2}^{T} \Upsilon_{2}\right) \Delta(k) \\
& -2 \bar{S}_{k}^{T}\left\{\left[\begin{array}{c}
\left(A-L \Gamma_{1}\right)^{T} P L \\
G^{T} P L \\
-\Gamma_{2}^{T} L^{T} P L \\
D^{T} P L
\end{array}\right]+\left[\begin{array}{c}
-\Gamma_{1}^{T} \Upsilon_{2}^{T} \\
0 \\
-\Gamma_{2}^{T} \Upsilon_{2}^{T} \\
\Upsilon_{1}^{T}
\end{array}\right] \Upsilon_{2}\right\} \Delta(k) \\
& +2 \bar{S}_{k}^{T}\left[\begin{array}{c}
-\Gamma_{1}^{T} \Upsilon_{2}^{T} \\
0 \\
-\Gamma_{2}^{T} \Upsilon_{2}^{T} \\
\Upsilon_{1}^{T}
\end{array}\right] \Delta F(k)<0
\end{aligned}
$$

where

$$
\begin{gathered}
\bar{\Psi}_{2}=\left[\begin{array}{cccc}
\bar{\Psi}_{3} & \left(A-L \Gamma_{1}\right)^{T} P G & \bar{\Psi}_{4} & \bar{\Psi}_{5} \\
* & -\lambda_{2}^{2} I+G^{T} P G & -G^{T} P L \Gamma_{2} & G^{T} P D \\
* & * & \bar{\Psi}_{6} & N_{1} \\
* & * & * & N_{2}
\end{array}\right] \\
N_{1}=-\Gamma_{2}^{T} L^{T} P D-M_{5} \\
N_{2}=D^{T} P D+M_{6} \\
\bar{\Psi}_{3}=\left(A-L \Gamma_{1}\right)^{T} P\left(A-L \Gamma_{1}\right)-P+\lambda_{2}^{2} U_{2}^{T} U_{2} \\
+\lambda_{1}^{2} E^{T} U_{1}^{T} U_{1} E+\Gamma_{1}^{T} \Upsilon_{2}^{T} \Upsilon_{2} \Gamma_{1} \\
\bar{\Psi}_{4}=-\left(A-L \Gamma_{1}\right)^{T} P L \Gamma_{2}+\Gamma_{1}^{T} \Upsilon_{2}^{T} \Upsilon_{2} \Gamma_{2} \\
\bar{\Psi}_{5}=\left(A-L \Gamma_{1}\right)^{T} P D-\Gamma_{1}^{T} \Upsilon_{2}^{T} \Upsilon_{1} \\
\bar{\Psi}_{6}=-\lambda_{1}^{2} I+\Gamma_{2}^{T} L^{T} P L \Gamma_{2}+\Gamma_{2}^{T} \Upsilon_{2}^{T} \Upsilon_{2} \Gamma_{2}
\end{gathered}
$$

Denote $R=P L$, it can be seen that

$$
\begin{aligned}
\Delta \Phi_{1} & =\bar{S}_{k}^{T} \bar{\Psi}_{2} \bar{S}_{k}+\Delta F^{T}(k) \Delta F(k)-2 \Delta F^{T}(k) \Upsilon_{2} \Delta(k) \\
& +\Delta^{T}(k)\left(R^{T} P^{-1} R+\Upsilon_{2}^{T} \Upsilon_{2}\right) \Delta(k) \\
& -2 \bar{S}_{k}^{T}\left\{\left[\begin{array}{c}
\left(A-L \Gamma_{1}\right)^{T} R \\
G^{T} R \\
-\Gamma_{2}^{T} L^{T} R \\
D^{T} R
\end{array}\right]+\left[\begin{array}{c}
-\Gamma_{1}^{T} \Upsilon_{2}^{T} \\
0 \\
-\Gamma_{2}^{T} \Upsilon_{2}^{T} \\
\Upsilon_{1}^{T}
\end{array}\right] \Upsilon_{2}\right\} \Delta(k) \\
& +2 \bar{S}_{k}^{T}\left[\begin{array}{c}
-\Gamma_{1}^{T} \Upsilon_{2}^{T} \\
0 \\
-\Gamma_{2}^{T} \Upsilon_{2}^{T} \\
\Upsilon_{1}^{T}
\end{array}\right] \Delta F(k)<0
\end{aligned}
$$

By using the Schur complement formula, (34) can be further reduced to

$$
\bar{\Psi}_{9}=\left[\begin{array}{cc}
\bar{\Psi}_{7} & \bar{\Psi}_{7}^{T} \\
\bar{\Psi}_{8} & -P
\end{array}\right]<0
$$




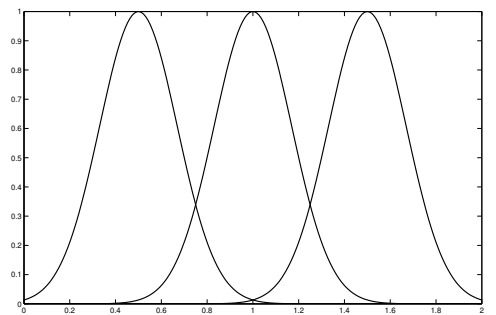

Fig. 1. Three basis functions for approximating the output PDF

where

$$
\bar{\Psi}_{7}=\left[\begin{array}{cccc}
\bar{\Psi}_{1} & 0 & \Gamma_{1}^{T} \Upsilon_{2}^{T} \Upsilon_{2} \Gamma_{2} & \Gamma_{1}^{T} \Upsilon_{2}^{T} \Upsilon_{1} \\
* & -\lambda_{2}^{2} I & 0 & 0 \\
* & * & -\lambda_{1}^{2} I+\Gamma_{2}^{T} \Upsilon_{2}^{T} \Upsilon_{2} \Gamma_{2} & -\Gamma_{2}^{T} \Upsilon_{2}^{T} \Upsilon_{1} \\
* & * & * & -I+\Upsilon_{1}^{T} \Upsilon_{1}
\end{array}\right]
$$

and

$$
\bar{\Psi}_{8}=\left[\begin{array}{llll}
P A-R \Gamma_{1} & P G & -R \Gamma_{2} & P D
\end{array}\right]
$$

which is equilevent to (30). If (30) holds, a positive scalar exists $\varrho_{1}$ so that $\bar{\Psi} \leq-\varrho_{1} I$. Thus, it can be seen that

$$
\begin{aligned}
\Delta \Phi_{1} & \leq-\varrho_{1}\left\|S_{k}\right\|^{2}-2\left\{\left\|\tilde{A}^{T} R\right\|+\left\|\tilde{T}^{T}\right\|\left\|\Upsilon_{2}\right\|\right\}\|\Delta(k)\|\left\|S_{k}\right\| \\
& -2\|\Delta F(k)\|\left\|\Upsilon_{2}\right\|\|\Delta(k)\|+2\left\|\tilde{T}^{T}\right\|\|\Delta F(k)\|\left\|S_{k}\right\| \\
& +\|\Delta(k)\|^{2}\left\{\left\|R^{T} P^{-1} R\right\|+\left\|\Upsilon_{2}\right\|^{2}\right\}+\|\Delta F(k)\|^{2} \\
& \leq-\varrho\left\|S_{k}\right\|^{2}-2\left\|\Xi_{1}\right\|\left\|S_{k}\right\|+\Xi_{2}
\end{aligned}
$$

where

$$
\begin{aligned}
& \tilde{A}=\left[\begin{array}{llll}
A-L \Gamma_{1} & P G & -R \Gamma_{2} & P D
\end{array}\right] \\
& \tilde{T}=\left[\begin{array}{llll}
-\Upsilon_{2} \Gamma_{1} & 0 & -\Upsilon_{2} \Gamma_{2} & \Upsilon_{2}
\end{array}\right] \\
& \Xi_{1}=\tilde{\delta}\left\{\left\|\tilde{A}^{T} R\right\|+\left\|\tilde{T}^{T}\right\|\left\|\Upsilon_{2}\right\|\right\}-\left\|\tilde{T}^{T}\right\|\|\Delta F(k)\|
\end{aligned}
$$

and

$$
\begin{aligned}
\Xi_{2} & =\|\Delta F(k)\|^{2}-2\|\Delta F(k)\|\left\|\Upsilon_{2}\right\| \tilde{\delta} \\
& +\tilde{\delta}^{2}\left\{\left\|R^{T} P^{-1} R\right\|+\left\|\Upsilon_{2}\right\|^{2}\right\}
\end{aligned}
$$

it can be shown that

$$
\left\|\bar{S}_{k}\right\| \geq \varrho_{1}^{-1}\left(\left\|\Xi_{1}\right\|+\sqrt{\left\|\Xi_{1}\right\|^{2}+\varrho_{1}\left\|\Xi_{2}\right\|}\right)
$$

which implies

$$
\left\|\bar{S}_{k}\right\| \leq \max \left\{\left\|\bar{S}_{k}(1)\right\|, \varrho_{1}^{-1}\left(\left\|\Xi_{1}\right\|+\sqrt{\left\|\Xi_{1}\right\|^{2}+\varrho_{1}\left\|\Xi_{2}\right\|}\right)\right\}
$$

This means that the error system in (28) is asymptotically stable under $\bar{\Psi}<0$.

\section{An Illustrated Example}

In this section, a simulation study of the proposed method will be described. First, the system model and RBF neural network components are introduced, and then the performance of the FDD will be investigated.

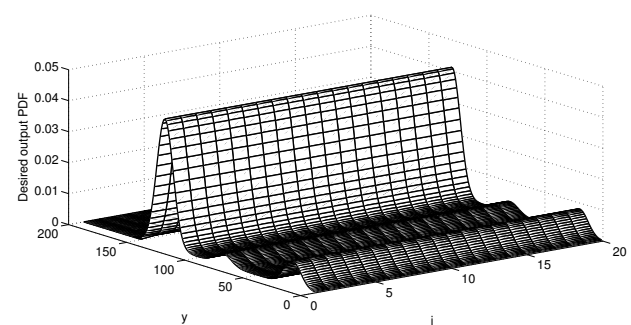

Fig. 2. Desired output PDF

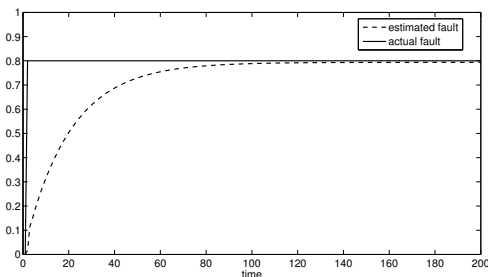

Fig. 3. Fault and its estimation under the filter

For a stochastic system with non-Gaussian process, it is supposed that the output PDF can be formulated by using three-layer neural network with three radial basis activation functions as shown in Fig. 1 with the following initial conditions over its definition interval $[a, b]$.

$$
\begin{gathered}
y \in[0,2], \\
\mu_{1}=0.5, \mu_{2}=1.0, \mu_{3}=1.5 \\
\sigma_{1}=\sigma_{2}=\sigma_{3}=0.2
\end{gathered}
$$

This would mean that the output PDF of the stochastic system is described as.

$$
\begin{aligned}
\sqrt{\gamma(y, u(k), F(k))} & =R(y) V(k)+r_{n}(y) h(V(k)) \\
& +\omega(y, u(k), F(k))
\end{aligned}
$$

where

$$
R(y)=\left[r_{1}(y), r_{2}(y)\right]
$$

and

$$
V(t)=\left[v_{1}(t), v_{2}\right]^{T}
$$

The weight vector behaves dynamically as described in (6) with the following parameters:

$$
\begin{gathered}
A=\left[\begin{array}{cc}
-0.45 & 0.03 \\
0.1 & -0.28
\end{array}\right], B=\left[\begin{array}{cc}
0.45 & 0.01 \\
0.01 & -0.86
\end{array}\right], \\
G=\left[\begin{array}{cc}
{[0.02} & 0 \\
0 & 0.01
\end{array}\right], D=E=\left[\begin{array}{ll}
1 & 0 \\
0 & 1
\end{array}\right]
\end{gathered}
$$

The nonlinear function was chosen as follows.

$$
g(V(t))=\left[\begin{array}{c}
0 \\
\sqrt{v_{1}^{2}+v_{2}^{2}}
\end{array}\right]
$$

The initial value of the weight vector is set as $V_{1}(0)=$ $[0.001,0.001]^{T}$. In addition, the matrices $U_{1}$ and $U_{2}$ were chosen as follows. 


$$
U_{1}=\left[\begin{array}{cc}
0.1 & 0 \\
0 & 0.1
\end{array}\right], U_{2}=\left[\begin{array}{ll}
1 & 0 \\
0 & 1
\end{array}\right],
$$

Assume that the modelling error satisfies $|\omega(y, u(k), F)| \leq 0.002$. The bound of medelling error satisfies $\tilde{\delta}=0.0008$ for $\mu(y)=1$. From (2), it can be compute that

$$
\begin{aligned}
\Lambda_{1} & =\left[\begin{array}{ll}
0.3010 & 0.0347 \\
0.0347 & 0.3010
\end{array}\right], \Lambda_{2}=\left[\begin{array}{ll}
0.0001 & 0.0350
\end{array}\right], \\
\Lambda_{3} & =0.3413
\end{aligned}
$$

Also, from (11), it can be seen that

$$
\Gamma_{1}=\left[\begin{array}{ll}
0.0389 & 0.0348
\end{array}\right], \Gamma_{2}=0.4225
$$

To demonstrate the effectiveness of the proposed algorithm, the fault is chosen to be a constant signal as $F(t)=0.8$, and it is supposed to commence at $T=2 s$.

Firstly, we consider the fault detection problem. Using Theorem 1 with $\lambda_{1}=\lambda_{2}=1$ it can be calculated that

$$
P=\left[\begin{array}{cc}
1.5941 & 0.1479 \\
0.1479 & 95.5499
\end{array}\right], R=\left[\begin{array}{l}
14.9186 \\
50.1447
\end{array}\right], L=\left[\begin{array}{l}
9.3113 \\
0.5104
\end{array}\right]
$$

Next, the fault diagnosis problem is considered for the above system and fault. Using Theorem 2, the following results can be obtained:

$$
\begin{gathered}
P=\left[\begin{array}{cc}
2.272 & -0.023 \\
-0.023 & 2.305
\end{array}\right], R=\left[\begin{array}{c}
-3.956 \\
0.920
\end{array}\right], L=\left[\begin{array}{c}
-1.737 \\
0.382
\end{array}\right] \\
\Upsilon_{1}=0.97, \Upsilon_{2}=1.3
\end{gathered}
$$

By applying the nonlinear fault isolation filter, Fig. 3 shows that such a filter can effectively diagnose the actuator fault.

\section{ACKNOWLEDGMENTS}

This work is jointly supported by the Chinese National Fundamental Research Program (No. 2009CB320604), the National Natural Science of China (No. 61074071,60534010), UK EPSRC (EP/I016643/1, EP/E050441/1-CICADA), the Funds for Creative Research Groups of China (No.60821063), and 111 Project (No. B08015).

\section{REFERENCES}

[1] K. Astrom, Introduction to Stochastic Control Theory. New York: Academic Press, 1970.

[2] B. Anderson and J. Moore, Linear Optimal Control. Englewood Cliffs, NJ: Prentice Hall, 1971.

[3] V. Solo, "Stochastic adaptive control and martingale limit theory," Automatic Control, IEEE Transactions on, vol. 35, no. 1, pp. 66-71, 1990.

[4] Y. Xia, P. Shi, G. Liu, and D. Rees, "Sliding mode control for stochastic jump systems with time-delay," in Intelligent Control and Automation, 2006. WCICA 2006. The Sixth World Congress on, vol. 1, pp. 354-358, 2006.

[5] H. Wang, Bounded dynamic Stochastic Systems: Modelling and Control. London: Springer-Verlag, 2000.

[6] H. Wang, "Robust control of the output probability density functions for multivariable stochastic systems with guaranteed stability," Automatic Control, IEEE Transactions on, vol. 44, no. 11, pp. 2103-2107, 1999.

[7] T. J. Crowley and K. Y. Choi, "Experimental studies on optimal molecular weight distribution control in a batch-free radical polymerization process," Chemical Engineering Science, vol. 53, no. 15, pp. 27692790, 1998.
[8] Y. Shibasaki, T. Araki, R. Nagahata, and M. Ueda, "Control of molecular weight distribution in polycondensation polymers 2 . poly(ether ketone) synthesis," European Polymer Journal, vol. 41, no. 10, pp. 2428 $2433,2005$.

[9] X. Sun, H. Yue, and H. Wang, "Modelling and control of the flame temperature distribution using probability density function shaping," Transactions of the Institute of Measurement and Control, vol. 28, no. 5, pp. 401-428, 2006.

[10] A. Wang, P. Afshar, and H. Wang, "Complex stochastic systems modelling and control via iterative machine learning," Neurocomputing, vol. 71 , no. 13-15, pp. 2685-2692, 2008.

[11] C. A. Dunbar and A. J. Hickey, "Evaluation of probability density functions to approximate particle size distributions of representative pharmaceutical aerosols," Journal of Aerosol Science, vol. 31, no. 7, pp. 813-831, 2000.

[12] D. Shi, N. H. El-Farra, M. Li, P. Mhaskar, and P. D. Christofides, "Predictive control of particle size distribution in particulate processes," Chemical Engineering Science, vol. 61, no. 1, pp. 268-281, 2006.

[13] A. Wang, H. Wang, and L. Guo, "Recent advances on stochastic distribution control: Probability density function control," in Control and Decision Conference, 2009. CCDC '09. Chinese, pp. xxxv-xli, 2009.

[14] L. Guo and H. Wang, "Pseudo-pid tracking control for a class of output pdfs of general non-gaussian stochastic systems," Processing of the 2003 American Control Conference, Denver, Colorado, USA, pp. 362-367, 2003

[15] Y. Wang and H. Wang, "Suboptimal mean controllers for bounded and dynamic stochastic distributions," Journal of Process Control, vol. 12, pp. 445-452, 2002

[16] H. Wang and H. Yue, "A rational spline model approximation and control of output probability density functions for dynamic stochastic systems," Transactions of the Institute of Measurement and Control, vol. 125, pp. 93-105, 2003.

[17] J. L. Zhou, H. Yue, and H. Wang, "Shaping of output pdf based on the rational square-root b-spline model," Acta Automatic Sinical, vol. 31, no. 3, pp. 343-351, 2005.

[18] H. Wang, "Multivariable output probability density function control for non-gaussian stochastic systems using simple mlp neural networks," Proceedings of the IFAC International Conference on Intelligent Control Systems and Signal Processing, Algarve, Portugal, pp. 84 89, 2003.

[19] H. Wang and P. Afshar, "Ilc-based fixed-structure controller design for output pdf shaping in stochastic systems using lmi techniques," IEEE transactions on automatic control, vol. 54, no. 4, pp. 760-773, 2009.

[20] R. Patton, P. Frank, and R. Clark, Fault diagnosis in dynamic systems. Theory and application. Englewood Cliffs, NJ: Prentice Hall, 1989.

[21] R. Isermann, "Fault diagnosis of machines via parameter estimation and knowledge processing tutorial paper," Automatica, vol. 29, no. 4 pp. 815-836, 1993 .

[22] H. Wang and W. Lin, "Applying observer based fdi techniques to detect faults in dynamic and bounded stochastic distributions," International Journal of Control, vol. 73, pp. 1424-1436, 2000.

[23] Y. M. Zhang, L. Guo, and H. Wang, "Filter-based fault detection and diagnosis using output pdfs for stochastic systems with time delays," International Journal of Adaptive Control and Signal Processing, vol. 20, no. 4, pp. 175-194, 2006.

[24] R. Isermann and P. Balle, "Trends in the application of model based fault detection and diagnosis of technical process," in Proceedings of the IFAC World Congress, pp. 1-12, 1996.

[25] M. Karny, I. Nagy, and J. Novovicova, "Mixed-data multi-modelling for fault detection and isolation," International Journal of Adaptive Control and Signal Processing, vol. 16, pp. 61-83, 2002.

[26] L. Guo and H. Wang, "Fault detection and diagnosis for general stochastic systems using b-spline expansions and nonlinear filters," IEEE Transactions on Circuits and System, vol. 52, no. 8, pp. 1644 $1652,2005$.

[27] H. Wang and P. Afshar, "Radial basis function based iterative learning control for stochastic distribution systems," Proceedings of the IEEE International Symposium on Intelligent Control, pp. 100-105, 2006.

[28] H. Wang, P. Afshar, and H. Yue, "Ilc-based generalised pi control for output pdf of stochastic systems using $\mathrm{lmi}$ and rbf neural networks," Proceedings of the IEEE Conference on Decision and Control, pp. 5048-5053, 2006. 